\title{
The Correlation Between COVID 19 and IUFD: Our Experience at a Tertiary Maternity Unit in France, the CHSF
}

\author{
Darido Jessie ${ }^{1,2, \text { *, Dussour Chloe }}{ }^{2}$, Lehrer Jade ${ }^{2}$, Grevoul Fesquet Julie ${ }^{2}$, EIHachem Larissa ${ }^{2}$, \\ Lavergne Delphine ${ }^{2}$, Khadam Louay ${ }^{2}$, Rigonnot Luc ${ }^{2}$ \\ ${ }^{1}$ Department of Obstetrics and Gynecology, Faculty of Medical Sciences, Lebanese University, Beirut, Lebanon \\ ${ }^{2}$ Department of Obstetrics and Gynecology, Centre Hospitalier Sud Francilien, Ile de France, France
}

Email address:

Jesydarido@hotmail.com (D. Jessie)

${ }^{*}$ Corresponding author

\section{To cite this article:}

Darido Jessie, Dussour Chloé, Grevoul Fesquet Julie, lehrer Jade, El Hachem Larissa, Lavergne Delphine, Rigonnot Luc. The Correlation Between COVID 19 and IUFD: Our Experience at a Tertiary Maternity Unit in France, the CHSF. Science Journal of Clinical Medicine. Vol. 9, No. 4, 2020, pp. 87-90. doi: 10.11648/j.sjcm.20200904.11

Received: September 7, 2020; Accepted: September 28, 2020; Published: October 16, 2020

\begin{abstract}
The data concerning this infection during pregnancy is still limited. Up until now there are no clear established correlations between the COVID 19 infection during pregnancy and its potential risks for the fetus including the stillbirth issue. At our center, the frequency of occurrence of stillbirths between January - June 2019 and January - June 2020 increased. Starting from this, we aim to see if there is upon the IUFD cases of 2020 any COVID 19 infection. Most of the patients refused to do the test due to various causes. 9 patients out of 20 accepted to do the serology test. Only one tested positive and all of the others tested negative. Note that half of the patients who had negative serology have other predisposing factors for stillbirth but the only patient who tested positive does not have any other risk factors. The question is whether it is cost effective or not to do the serology test of COVID systematically in the case of pregnant patients where stillbirth occurred? Further research concerning COVID infection in pregnancy is needed. This study will be enlarged and completed with another one that will include almost nine centers at Ile de France.
\end{abstract}

Keywords: Covid19, Pregnancy, Stillbirths, Serology Test, Fetal Outcome, Obstetrical Complications

\section{Introduction}

Corona viruses (COV) are non-segmented enveloped viruses that are divided into two main large groups:

1) The Human Coronaviruses (alpha)

2) The Zoonotic Coronaviruses (Beta)

Both of them are simple stranded RNA viruses.

Historically, COV were revealed via:

1) The Middle East Respiratory Syndrome (MERS) that appeared in the Arabian Peninsula in 2012. It provoked $10 \%$ of mortality and was characterized by low infectiousity;

2) The Severe Acute Respiratory Syndrome (SARS) that appeared in China in 2002. It provoked $35 \%$ of mortality and had low infectiousity

Nowadays, we have the new Beta coronavirus, theSARSCOV2. Its sequence, as it is studied, revealed some similarities with the MERS-COV (79\%) and SARS-COV $(50 \%)$ [1]. Known as COVID-19, this new version of coronaviruses was highly infectious, particularly during the asymptomatic period of incubation, with a low rate of consequent mortality. Therefore, it kept spreading, became pandemic and resulted in a worldwide health crisis of rapid evolution with consequent socioeconomic effect due to international strict measurements of lockdowns and border closures. 3.0 million people were infected and more than 200,000 consequent mortalities $[2,3]$. Up until the $18^{\text {th }}$ of June, the total number of reported coronavirus mortality cases in France was 29603 (source: statista).

Pregnant women are affected by this virusat the different stages of pregnancy (first, second and third trimester) [4]. These impacts are subjected to multiple discussions. The SARS and MERS classical coronaviruses epidemics were responsible of many adverse fetal outcomes. What about the novel coronavirus COVID 19? COVID 19 and stillbirth is 
one of the questions that are raised [3]:

The data concerning this infection during pregnancy is still limited. Up until now there are no clear established correlations between the COVID 19 infection during pregnancy and its potential risks for the fetus including the stillbirth issue. Moreover, the reported cases of stillbirth on infected pregnant patients are limited. Severe maternal cases, as well as prematurity, fetal distress and stillbirth among newborns have been reported. However, these reported cases are still very limited. Note that the SARS-COV2 pandemic affected the prenatal management and surveillance of pregnancies worldwide.

\section{Objectives and Methods}

At our center, the frequency of occurrence of stillbirths between January - June 2019 and January - June 2020 increased. Starting from this, we aim through this to see if there is upon the IUFD cases of 2020 any COVID 19 infection and based on the results to proceed into affirming if there is a necessity to calculate the correlation between the two entities.

Based on the codes of registered IUFDs, we have selected all the patients in the considered period of time. The fetal mortality rates which is calculated via the number of stillbirths over the live births auditioned to the stillbirths. In addition, all the women that were included and have their stillbirth during 2020 were called asking for their contribution to do the COVID serology and to send their results via email, in order to avoid their return into a location that may have bad memory for them. Past medical history was taken from their medical record to see if there is any additional factors that may have interfered with the stillbirth For the adequacy of the reported information, the patients were asked on the phone if there was any decreased fetal movement and if they were under confinement or not. There are no uniform criteria for the definition of fetal death. However, in the hereby reported stillbirths the cutoff was considered starting from 20 weeks of gestation and above especially that the gestational age in these cases is known [5]. Note that these stillbirths occurred without any medical interruption of the pregnancy. All ethical considerations were taken in this procedure.

\section{Results}

The calculated values of fetal mortality rates for the considered two periods were compared in table 1 .

Table 1. Total of deliveries and stillbirths in 2019 and 2020.

\begin{tabular}{lll}
\hline & January till June 2019 & January till June 2020 \\
\hline Deliveries & 2517 & 2582 \\
Stillbirths & 11 & 20 \\
Rate $(\%)$ & 0.43 & 0.77 \\
\hline
\end{tabular}

The multiple risk factors that may interfere with the occurrence of IUFD are cited in Table 2.
Table 2. The risk factors of IUFD as mentioned by Rosenstein M and al [6].

\begin{tabular}{ll}
\hline Risk factors & N \\
\hline Multiple Gestations & Twins=1Triplets=1 \\
Growth restriction & None \\
Past Obstetric History & Previous still birth=1 \\
Decreased fetal movement & All of them \\
Maternal Age $<15$ years and $>$ 35 years & 3 \\
Comorbid Medical Conditions & Preeclampsia with RPH and \\
Late term pregnancy greater than 41 weeks & DIC=1GD=2 \\
ART & None \\
Lupus, renal disease, cholestasis of & antiphospholipid syndrome=1 \\
pregnancy & None \\
Smoking & \\
\hline
\end{tabular}

Table 3. The repartition of the results based on the presence or not of associated Risk factors.

\begin{tabular}{lllll}
\hline $\begin{array}{l}\text { PMH or } \\
\text { RF }\end{array}$ & $\begin{array}{l}\text { Refused } \\
\text { Serology\%(n) }\end{array}$ & $\begin{array}{l}\text { Negative\% } \\
(\mathbf{n})\end{array}$ & Positive\%(n) & Total \\
\hline With & $20(\mathrm{n}=4)$ & $20(\mathrm{n}=4)$ & $0(\mathrm{n}=0)$ & $40(\mathrm{n}=8)$ \\
Without & $35(\mathrm{n}=7)$ & $20(\mathrm{n}=4)$ & $5(\mathrm{n}=1)$ & $60(\mathrm{n}=12)$ \\
Total $(\%)$ & $55(\mathrm{n}=11)$ & $40(\mathrm{n}=8)$ & $5(\mathrm{n}=1)$ & $100 \mathrm{n}=20$ \\
\hline
\end{tabular}

Most of the patients refused to do the test (55\%) (Table 3) due to different causes: those with known predisposing risk factors considered that they already know the cause of IUFD and they do not need further test while the others that do not have any predisposing factor, have the socioeconomic causes mainly (expensive serology test, cannot leave work or children at home, etc).

9 patients out of 20 accepted to do the serology test. Only one tested positive and all of the others tested negative. The repartition of the results out of 20 pregnancies complicated with an IUFD came as follow: $55 \%$ refused to do the serology test, $40 \%$ were negative and $5 \%$ were positive (Figure 1).

Note that half of the patients who had negative serology have other predisposing factors for stillbirth but the only patient who tested positive does not have any other risk factors.

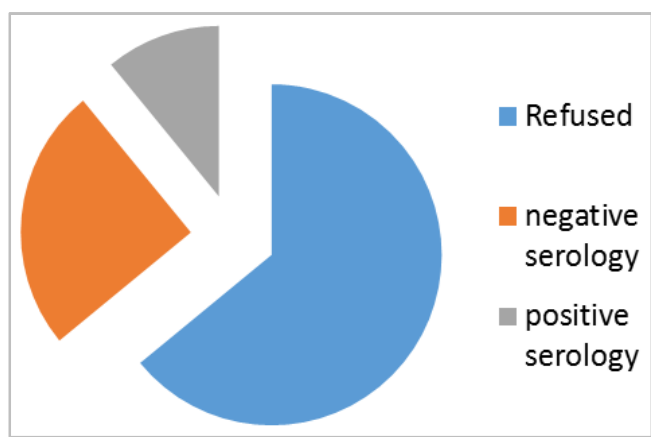

Figure 1. The results ef COVID serology over the 20 pregnant patients with IUFD.

\section{Discussion}

The impact of COVID 19 on pregnancy cannot be negligible especially if the maternal and fetal complications due to other types of coronaviruses in previous epidemics are 
taken into consideration [7]. It is evident that the sociodemographic characteristics and presupposed risk factors are of high importance.

In a rapid review on COVID - 19 infection in pregnancy, conducted by Mullins E et al, they found only one case of stillbirth over 29 delivered pregnant COVID 19 women affected by coronavirus during pregnancy [8]. Yang $Z$ et al reported a percentage of 1.2 percent regarding the fetal stillbirth outcome in pregnancies affected by COVID 19 only in their third trimester [9]. Another literature systematic review, there was one stillbirth out of 48 deliveries [10]. Knight $M$ et al leaded a national Cohort study in UK concerning the characteristics and outcomes of pregnant women admitted to hospital with confirmed SARS-CoV-2: Out of the 262 women who had given birth, three were stillborn [11]. 8 deliveries out of 46 pregnant patients with COVID 19 infection in Washington State occurred with one stillbirth of unknown etiology [12].

However, in the cohort of Pierce-Williams et al out of the 32 pregnant women that delivered while they were infected with COVID 19 There were no stillbirths [13].

On the other hand, many reported cases of viral infection during pregnancy were associated with stillbirth so it is very eligible to ask the correlation between coronavirus and stillbirths [14].

Unfortunately, in our serie this correlation cannot be neither confirmed nor rejected. In fact we did not test the serology in another comparative serie of pregnant patients with a normal course of pregnancy to see the percentage of COVID. This has many major limitations reflected by the percentage of patients in our serie that refused to do the test.

\section{Conclusion}

The question is whether it is cost effective or not to do the serology test of COVID systematically in all pregnant patients especially that the correlation is not confirmed.

Further research concerning COVID infection in pregnancy along with the possible consequent fetal and neonatal complications may be effective into modifying the protocols of management of such cases that are up till now based on the obstetrical indication for delivery if the maternal respiratory condition is stable. Maybe more monitoring is required at the level of the suspected cases.

Our study will be enlarged and completed with another one that will include almost 9 centers at Ile de France so we can clarify if the correlation between the viral infection and stillbirth is significant or not.

\section{Funding}

No resources

\section{Availability of Data and Materials}

Medline databases

\section{Authors' Contributions}

Darido Jessie wrote the first draft and all authors read and approved the final manuscript for publication.

\section{Conflict of Interest}

All the authors do not have any possible conflicts of interest.

\section{Consent for Publication}

The needed consent was taken from all the patients.

\section{Ethics Approval and Consent to Participate}

This article responds to the ethical basics of research.

\section{Acknowledgements}

We are grateful to Dr Chloe Dussour for proofreading and editing this article.

\section{References}

[1] Li X, GengM, Peng Y, MengL, LuS, Molecular immune pathogenesis and diagnosis of COVID-19, Journal of Pharmaceutical Analysis, Volume 10, Issue 2, April 2020, Pages 10-108.

[2] Pierce-Williams RAM, Burd J, Felder L, et al. Clinical course of severe and critical COVID-19 in hospitalized pregnancies: a US cohort study [published online ahead of print, 2020 May 8]. Am J Obstet Gynecol MFM. 2020; 100134. doi: 10.1016/j.ajogmf.2020.100134.

[3] Lambelet V, Vouga M, Pomar L, et al. Sars-CoV-2 in the context of past coronaviruses epidemics: Consideration for prenatal care [published online ahead of print, 2020 May 26]. PrenatDiagn. 2020; 10.1002/pd.5759. doi: 10.1002/pd.5759.

[4] Huijun Chen, Juanjuan Guo, Chen Wang, Fan Luo, Xuechen Yu, Wei Zhang, Jiafu Li, Dongchi Zhao, Dan Xu, Qing Gong, Jing Liao, Huixia Yang, Wei Hou, Yuanzhen Zhang, Clinical characteristics and intrauterine vertical transmission potential of COVID-19 infection in nine pregnant women: a retrospective review of medical records, The Lancet, Volume 395, Issue 10226, 7-13 March 2020, Pages 760-762.

[5] National Center for Health Statistics. Model state vital statistics act and regulations. Atlanta, GA: Centers for Disease Control and Prevention; 1992. Available at: https://www.cdc.gov/nchs/data/misc/mvsact92b.pdf. Retrieved September 16, 2019.

[6] Rosenstein MG, Snowden JM, Cheng YW, Caughey AB the mortality risk of expectant management compared with delivery stratified by gestational age and race and ethnicity. Am J obstetgynecol 2014; 211: 660; e1-8.

[7] Masmejan S, pomar L, Lepigeon K, Favre G, Baud D, Rieder W. COVID 19 et grossesse (COVID 19 and pregnancy). Rev Med Suisse. 2020; 16 (692): 944-946. 
[8] Mullins E, Evans D, Viner RM, O'Brien P, Morris E. Coronavirus in pregnancy and delivery: rapid review. Ultrasound Obstet Gynecol. 2020; 55 (5): 586-592. doi: 10.1002/uog.22014.

[9] Yang Z, Wang M, Zhu Z, Liu Y. Coronavirus disease 2019 (COVID-19) and pregnancy: a systematic review [published online ahead of print, 2020 Apr 30]. J Matern Fetal Neonatal Med. 2020; 1-4. doi: 10.1080/14767058.2020.1759541.

[10] Della Gatta AN, Rizzo R, Pilu G, Simonazzi G. Coronavirus disease 2019 during pregnancy: a systematic review of reported cases. Am J Obstet Gynecol. 2020; 223 (1): 36-41. doi: 10.1016/j.ajog.2020.04.013.

[11] Knight M, Bunch K, Vousden N, et al. Characteristics and outcomes of pregnant women admitted to hospital with confirmed SARS-CoV-2 infection in UK: national population based cohort study. BMJ. 2020; 369: m2107. Published 2020 Jun 8. doi: 10.1136/bmj.m2107.
[12] Lokken EM, Walker CL, Delaney S, et al. Clinical Characteristics of 46 Pregnant Women with a SARS-CoV-2 Infection in Washington State [published online ahead of print, 2020 May 18]. Am J Obstet Gynecol. 2020; S0002-9378 (20) 30558-5. doi: 10.1016/j.ajog.2020.05.031.

[13] Pierce-Williams RAM, Burd J, Felder L, et al. Clinical course of severe and critical COVID-19 in hospitalized pregnancies: a US cohort study [published online ahead of print, 2020 May 8]. Am J Obstet Gynecol MFM. 2020; 100134. doi: 10.1016/j.ajogmf.2020.100134.

[14] EC, Tirado V, Lopes KD, et al. Zika virus infection in pregnancy: a systematic review of disease course and complications. Reprod Health. 2017; 14 (1): 28. Published 2017 Feb 28. doi: 10.1186/s12978-017-028. 